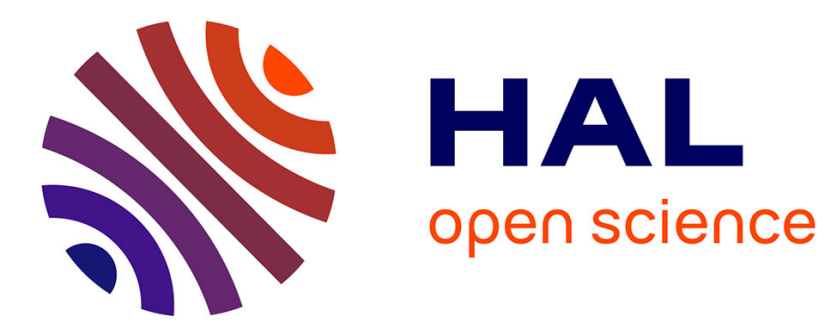

\title{
Observability of systems when measurements are not time-stamped
}

Eric Busvelle

\section{To cite this version:}

Eric Busvelle. Observability of systems when measurements are not time-stamped. 2021 9th International Conference on Systems and Control (ICSC), Nov 2021, Caen, France. pp.264-267, 10.1109/ICSC50472.2021.9666600 . hal-03582401

\section{HAL Id: hal-03582401 \\ https://hal.science/hal-03582401}

Submitted on 21 Feb 2022

HAL is a multi-disciplinary open access archive for the deposit and dissemination of scientific research documents, whether they are published or not. The documents may come from teaching and research institutions in France or abroad, or from public or private research centers.
L'archive ouverte pluridisciplinaire HAL, est destinée au dépôt et à la diffusion de documents scientifiques de niveau recherche, publiés ou non, émanant des établissements d'enseignement et de recherche français ou étrangers, des laboratoires publics ou privés. 


\title{
Observability of systems when measurements are not time-stamped
}

\author{
Éric Busvelle ${ }^{1}$
}

\begin{abstract}
In this paper, we define observability when the measurements are not time-stamped. In general, observer theory always assumes that the outputs correspond to correctly time-stamped states (although varying delays are sometimes considered). In practice, this is not always the case. The extreme situation is the complete absence of a time stamp. In this paper, we propose a definition that we will justify by a few examples. We try to characterize the rarity of systems with unknown dating but which are nevertheless observable.
\end{abstract}

\section{INTRODUCTION}

Many papers deal with observability under asynchronous sampling [5], [6], [8], [13] or sampling with delay [5], [8], [13] and even in the presence of unknown state delays [10] but to our knowledge, there are no papers dealing with observability under unknown or at least inaccurate sampling. However, the problem may arise in many situations and, in order to illustrate our purpose, we will present two examples. The first one is a very well-known game used to teach sequencing skills to children, see Figure 1. If one considers each image as a measurement, this particular example illustrates the fact that

- it is possible to retrieve the order, thanks to the model

- it is also possible to retrieve some information concerning the actual trajectory of the ball, although dating of pictures is unknown

Let is considering a second and more mathematical example. We want to retrieve the trajectory of an object moving in the space. Observations consist of several photos of the object but unfortunately these photos are not correctly timestamped.

This problem may occur in several cases : the camera may be a webcam, image may arrive thanks to a network with a poor quality of service (such as ethernet with UDP frames...) or even with an accurate camera but because of a rapid motion and the rolling shutter effect. In the more difficult case, we have several pictures of the motion but without any idea of their time stamp. The question is: what can we get from the measurements, or more precisely, is the system observable?

The quick answer to this question is no. Indeed, without any information concerning absolute time, we cannot expect to retrieve the initial state of the system since we have no idea of initial time. Let us consider a simple example to make following definitions more intuitive. Later, we will refer to this example as the case of the ball in a vertical plane.

We consider the case of a ball moving in a vertical plane, and we denote $(x, z)$ the position of the ball, $x$ being the

\footnotetext{
1 LIS, UMR CNRS 7020, Université de Toulon, Aix Marseille Univ., France busvelledlis-lab.fr
}

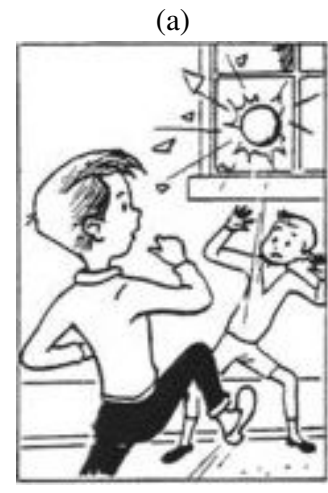

(c)

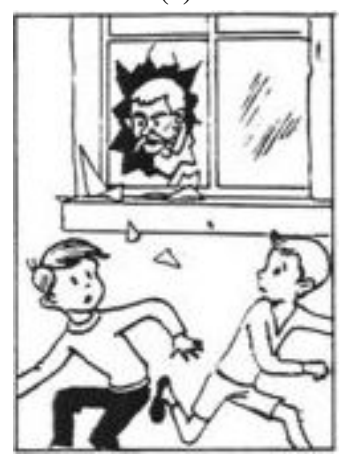

(b)

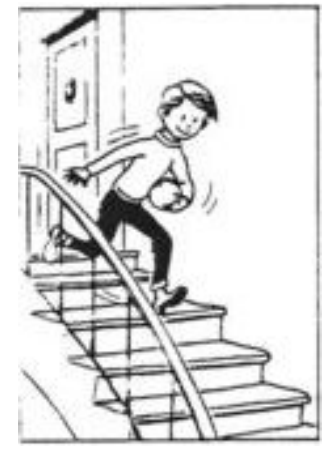

(d)

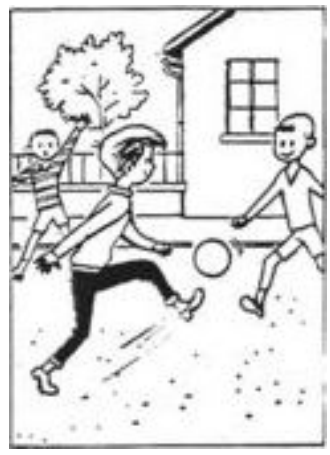

Fig. 1: Arrange all pictures according to the chronological order

coordinate along the horizontal axe and $z$ being the coordinate along the vertical axe. The model is then $\ddot{x}=0$ and $\ddot{z}=-g$ (gravity). The trajectory is the solution of a linear system in the phase space $\mathbb{R}^{4}$, precisely $x(t)=v_{x} t+x_{0}$ and $z(t)=-\frac{1}{2} g t^{2}+v_{z} t+z_{0}$, where $x_{0}, z_{0}, v_{x}$ and $v_{z}$ are the initial conditions. We assume that $v_{x}>0$ and we will come back to this assumption.

Measurements consists in some pictures of the ball, the optical axis being orthogonal to the plane of the trajectory. Therefore, each measurement $y_{i}$ is given by $y_{i}=$ $\left(x\left(t_{i}\right), z\left(t_{i}\right)\right), t_{i}$ being unknown. Clearly, totally unknown time stamp is an academic situation, but one can consider inaccurate time stamp as an actual case. Now let's try to figure out what kind of observability we can expect. Using the measurements in $\mathbb{R}^{2}$, we can eliminate $t_{i}=\frac{x\left(t_{i}\right)-x_{0}}{v_{x}}$, hence

$$
\begin{array}{r}
z\left(t_{i}\right)=-\frac{1}{2} \frac{g}{v_{x}^{2}} x\left(t_{i}\right)^{2}+\left(\frac{g x_{0}}{v_{x}^{2}}+\frac{v_{z}}{v_{x}}\right) x\left(t_{i}\right) \\
+\left(z_{0}-\frac{1}{2} g \frac{x_{0}^{2}}{v_{x}^{2}}-x_{0} \frac{v_{z}}{v_{x}}\right)
\end{array}
$$


which means that the two components of each measurement are located on a parabola (the trajectory parameterized in time). $x_{0}$ and $z_{0}$ are unobservable because they refer to an absolute time. We can decide to refer to $x\left(t_{0}\right)$ and $z\left(t_{0}\right)$ (the first measurement) as the initial state. Setting $x_{0}=x\left(t_{0}\right)$ and $z_{0}=z\left(t_{0}\right)$, it remains to retrieve the initial value of the speed of the ball. Using three more measurements, we estimate the trajectory and hence the coefficients of the parabola. The coefficient of the square gives $v_{x}^{2}$ and hence $v_{x}$ since $v_{x}>0$ and the other ones give $v_{z}$.

Therefore, the system may be considered as observable modulo a time translation along the trajectory. More concretely, it is possible to retrieve the whole trajectory but also the position along the trajectory of (for instance) the first measurement. This second property is important, as we will see later on.

To be more complete considering this same example, let us relax the hypothesis $v_{x}>0$. Then it is clear that in the hypothesis of totally unknown sampling, the sign of $v_{x}$ is still unobservable. But even if we suppose that the sampling is only inaccurate, the case $v_{x}=0$ remains a problem since in this case, $v_{z}$ is unobservable (since all measurements are located on the same line).

\section{DEFINITIONS}

We consider a general smooth nonlinear system $\frac{d x(t)}{d t}=$ $f(x(t))$ where $x(t)$ belongs to a $n$-dimensional manifold $\mathcal{X}$, and $f$ is a smooth vector field (in this paper, smooth means $\left.C^{\infty}\right)$. Let us denote $x(0)=x^{0}$. This system is referred as state equations. In the following, we will consider this system on interval $[0, T]$ and we suppose implicitly that $T<T_{e}$ where $T_{e}$ is the explosion time (maybe equal to $+\infty$ ).

We also consider a measurement smooth function $h$ from $\mathcal{X}$ into $\mathbb{R}^{p}$. We denote $S_{\mathcal{X}, p}$ the set of all these systems $\Sigma=(f, h)$. We suppose that measurements are available at $N$ sampling times $t_{1}, t_{2}, \cdots t_{N}$ in the interval $\left.] 0, T\right] \subset \mathbb{R}$, $t_{i} \neq t_{j}$ for $i \neq j$. Classical continuous-discrete observability is defined as the injectivity of the function $x^{0} \longrightarrow$ $\left(h\left(x\left(t_{1}\right)\right), \ldots, h\left(x\left(t_{N}\right)\right)\right)$. Here, continuous stands for the continuous time of the state equations, discrete stands for the discrete-time measurements. Roughly speaking, observability is the property that the $N$ outputs are sufficient to determine the initial state of the system.

Usually, sample times $t_{1}, \ldots, t_{N}$ are known. As already explained in the introduction of this paper, we will suppose that time stamp of measurements are unknown. Let us define more precisely our framework. We set $y(k)=h\left(x\left(t_{k}\right)\right)$, $k=1, \ldots, N$ and we define the function

$$
P_{\Sigma}^{N}\left(x^{0}, t_{1}, \ldots, t_{N}\right)=(y(1), \ldots, y(N))
$$

from $\mathcal{X} \times \mathcal{T}^{N}$ into $\mathbb{R}^{N p}$ where $\mathcal{T}^{N}=\left\{\left(t_{1}, \ldots, t_{N}\right) \in\right.$ ] $\left.0, T]^{N} ; \quad t_{i} \neq t_{j} \quad \forall i \neq j\right\}$.

Let us denote by $F$ the flow application, defined by $F^{t}\left(x^{0}\right)=x(t)$, the solution of the differential equation at time $t$ when $x(0)=x^{0}$. Clearly, for any $0<\tau<t_{1}$ :

$$
P_{\Sigma}^{N}\left(x^{0}, t_{1}, \ldots, t_{N}\right)=P_{\Sigma}^{N}\left(F^{\tau}\left(x^{0}\right), t_{1}-\tau, \ldots, t_{N}-\tau\right)
$$

and this prevents us from defining observability under unknown sampling as the injectivity of $P_{\Sigma}^{N}\left(x^{0}\right.$ and $F^{\tau}\left(x^{0}\right)$ are indistinguishable). In fact, since sampling times are unknown, we can expect to retrieve the trajectory $x(t)$ but not precisely $x^{0}$ since we have no time reference (as in the example of the ball in a vertical plane, Page 1). The following definition is a little bit more restrictive definition of observability under unknown sampling.

Definition 1: System $\Sigma$ is observable under unknown sampling if, for $x^{0}, z^{0} \in \mathcal{X},\left(t_{k}\right)_{k=0, \ldots, N-1} \in \mathcal{T}^{N}$, $\left(s_{k}\right)_{k=0, \ldots, N-1} \in \mathcal{T}^{N}$

$$
P_{\Sigma}^{N}\left(x^{0}, t_{0}, \ldots, t_{N-1}\right)=P_{\Sigma}^{N}\left(z^{0}, s_{0}, \ldots, s_{N-1}\right)
$$

implies $F^{t_{0}}\left(x^{0}\right)=F^{s_{0}}\left(z^{0}\right)$.

In other words, knowing $y(1), \ldots, y(N)$ allows us to determine $x\left(t_{0}\right)$ ( $t_{0}$ being unknown).

Example 1: In order to illustrate the problem and our definitions, let us consider the following system

$$
\left\{\begin{array}{l}
\dot{x}_{1}=x_{2} \\
\dot{x}_{2}=x_{3} \\
\dot{x}_{3}=1
\end{array}\right.
$$

Clearly, this system admits the following solution

$$
\left\{\begin{array}{l}
x_{1}=x_{1}^{0}+x_{2}^{0} t+\frac{1}{2} x_{3}^{0} t^{2}+\frac{1}{6} t^{3} \\
x_{2}=x_{2}^{0}+x_{3}^{0} t+\frac{1}{2} t^{2} \\
x_{3}=x_{3}^{0}+t
\end{array}\right.
$$

- If $h(x)=x_{1}$, the system is clearly observable (the linear system is in a canonical form of observability). It is not observable under unknown sampling. For instance, $x=(0,0,0)$ and $z=(0,1,0)$ are indistinguishable. Indeed,

$$
\begin{aligned}
P_{\Sigma}^{N}\left((0,0,0), t_{1}, \ldots, t_{N-1}\right) & \\
= & \left(0, \frac{1}{6} t_{1}^{3}, \ldots, \frac{1}{6} t_{N-1}^{3}\right)
\end{aligned}
$$

and

$$
\begin{aligned}
& P_{\Sigma}^{N}\left((0,1,0), s_{1}, \ldots, s_{N-1}\right) \\
& \quad=\left(0, s_{1}+\frac{1}{6} s_{1}^{3}, \ldots, s_{N-1}+\frac{1}{6} s_{N-1}^{3}\right)
\end{aligned}
$$

which are equal as soon as $s_{j}$ for $j=1, \ldots, N-1$ are solutions in $] 0, T]$ of $s_{j}+\frac{1}{6} s_{j}^{3}=\frac{1}{6} t_{j}^{3}$ which is always possible for any $\left.\left.t_{j} \in\right] 0, T\right]$.

- If $h(x)=\left(x_{1}, x_{3}\right)$, the system is still observable and it remains observable under unknown sampling. Indeed, $P_{\Sigma}^{2}(x, t)=\left(x_{1}^{0}, x_{3}^{0}, x_{1}^{0}+x_{2}^{0} t+\frac{1}{2} x_{3}^{0} t^{2}+\frac{1}{6} t^{3}, x_{3}^{0}+t\right)$ which is clearly sufficient to determine $x_{1}^{0}, x_{2}^{0}$ and $x_{3}^{0}$.

Remark 1: We could have imagined another definition of observability under unknown sampling by asking, instead of Definition 1:

$$
\begin{aligned}
P_{\Sigma}^{N}\left(x^{0}, t_{0}, \ldots, t_{N-1}\right)=P_{\Sigma}^{N} & \left(z^{0}, s_{0}, \ldots, s_{N-1}\right) \\
& \Longrightarrow \exists \tau \quad z^{0}=F^{\tau}\left(x^{0}\right)
\end{aligned}
$$

meaning that measures are sufficient to identify the trajectory in the state space and nothing about the state at the first 
sample (see the example of the ball in a vertical plane, Page 1). This definition would be less restrictive since $F^{t}\left(x^{0}\right)=F^{s}\left(z^{0}\right)$ implies $F^{-s} F^{t}\left(x^{0}\right)=z^{0}=F^{\tau}\left(x^{0}\right)$ for $\tau=t-s$. The two definitions are not equivalent, but above all, an unobservable system could become observable with unknown sampling, as the following example shows. So definition 1 seems more appropriate.

Example 2: Let us consider the following system

$$
\left\{\begin{aligned}
\dot{x}_{1} & =x_{2} \\
\dot{x}_{2} & =-x_{1} \\
y & =h\left(x_{1}, x_{2}\right)=x_{1}^{2}+x_{2}^{2}
\end{aligned}\right.
$$

The solution being $x_{1}(t)=y \cos (t+\varphi)$ and $x_{2}(t)=$ $y \sin (t+\varphi)$ where $y$ is the (constant) output and $\varphi$ is not observable. Therefore, this system is not observable, and it is not observable under unknown sampling in the sense of Definition 1, although it satisfies the definition in Remark 1.

It is worth remarking that if we replace the single output by $h\left(x_{1}, x_{2}\right)=\left(x_{1}^{2}+x_{2}^{2}, x_{1}\right)$ then the system becomes observable in the classical sense but is still unobservable for unknown sampling in the sense of Definition 1 (the sign of $x_{2}^{0}$ remains undefined).

As we could imagine, we have the following result :

Proposition 2: If $\Sigma$ is observable under unknown sampling then it is observable.

Proof: It is sufficient to verify that there is no undistinguishable points. Indeed, thanks to Definition 1 , for known time stamp $t_{0}, t_{1}, \ldots t_{N-1}$, we have $P_{\Sigma}^{N}\left(x^{0}, t_{0}, \ldots, t_{N-1}\right)=P_{\Sigma}^{N}\left(z^{0}, t_{0}, \ldots, t_{N-1}\right)$, hence $F^{t_{0}}\left(x^{0}\right)=F^{t_{0}}\left(z^{0}\right)$ and equivalently $x^{0}=z^{0}$.

\section{MAIN RESULT}

Definition 1 is intuitive but not very practical for theoretical developments. Let us denotes

$$
\begin{aligned}
& \bar{P}_{\Sigma}^{N}(x, z, \bar{t}, \bar{s}) \\
&=\left(h(x), h\left(F^{t_{1}}(x)\right), \ldots, h\left(F^{t_{N-1}}(x)\right),\right. \\
&\left.h(z), h\left(F^{s_{1}}(z)\right), \ldots, h\left(F^{s_{N-1}}(z)\right)\right)
\end{aligned}
$$

where $x, z \in \mathcal{X}$ and $\bar{t}=\left(t_{k}\right)_{k=1, \ldots, N-1} \in \mathcal{T}^{N-1}, \bar{s}=$ $\left(s_{k}\right)_{k=1, \ldots, N-1} \in \mathcal{T}^{N-1}$

Because of (2), Definition 1 is equivalent to say that system $\Sigma$ is observable under unknown sampling if $\bar{P}_{\Sigma}^{N}$ : $\mathcal{X} \times \mathcal{X} \times \mathcal{T}^{N-1} \times \mathcal{T}^{N-1} \longrightarrow \mathbb{R}^{N p} \times \mathbb{R}^{N p}$ is such that

$$
\bar{P}_{\Sigma}^{N}(x, z, \bar{t}, \bar{s}) \in \Delta\left(\mathbb{R}^{N p} \times \mathbb{R}^{N p}\right) \Rightarrow(x, z) \in \Delta(\mathcal{X} \times \mathcal{X})
$$

or equivalently

$$
\begin{aligned}
& \bar{P}_{\Sigma}^{N}\left(\Delta(\mathcal{X} \times \mathcal{X})^{c} \times \mathcal{T}^{N-1} \times \mathcal{T}^{N-1}\right) \\
& \bigcap \Delta\left(\mathbb{R}^{N p} \times \mathbb{R}^{N p}\right)=\varnothing
\end{aligned}
$$

where $\Delta(A \times A)$ is the diagonal of $A \times A$ and $\Delta(A \times A)^{c}=$ $A \times A \backslash \Delta(A \times A)$ its complementary.

This is equivalent to $\bar{P}_{\Sigma}^{N} \pitchfork \Delta(\mathcal{X} \times \mathcal{X})$ because

$$
\begin{array}{r}
\operatorname{codim}\left(\Delta\left(\mathbb{R}^{N p} \times \mathbb{R}^{N p}\right)\right)=N p>2 n+2(N-1) \\
=\operatorname{dim}\left(\Delta(\mathcal{X} \times \mathcal{X})^{c} \times \mathcal{T}^{N-1} \times \mathcal{T}^{N-1}\right)
\end{array}
$$

as soon as $p \geq 3$ and $N$ large enough.

Using transversality theorem (see [1] or [9]), we proved the following result :

Theorem 3: The set of systems $\Sigma=(f, h) \in S_{\mathcal{X}, p}$ where $h$ is a function from $\mathcal{X}$ into $\mathbb{R}^{p}, p \geq 3$, that are observable under unknown sampling is residual in $S_{\mathcal{X}, p}$ w.r.t. $C^{\infty}$ topology.

Theorem 3 states that the property to be observable under unknown sampling is generic for systems where measurements have dimension greater or equal to 3 .

A question may arise : is this theorem true if $p=2$ ? To prove that it is not the case, let us consider a system whose state space is in $\mathbb{R}^{3}$ and suppose $p=2$. To make our system more concrete (without loss of generality, thanks to a change of coordinates) $h$ is chosen as the projection of the state on the plane made up of the first two variables. Let us choose a point of self-intersection of the projection of the trajectory on the plane. It may not exist, so we have to choose a system that is complicated enough for such points to exist (Lorenz system is clearly complicated enough but very simple linear systems are convenient, see e.g. Figure 2). It is well known that the existence of the self-intersection point is a stable property up to a small perturbation of the system, so there exists a neighborhood of $\Sigma=(f, h)$ such that there always exist two points $x \neq z$ in $\mathbb{R}^{3}$ such that $h(x)=h(z)=y_{0}$.

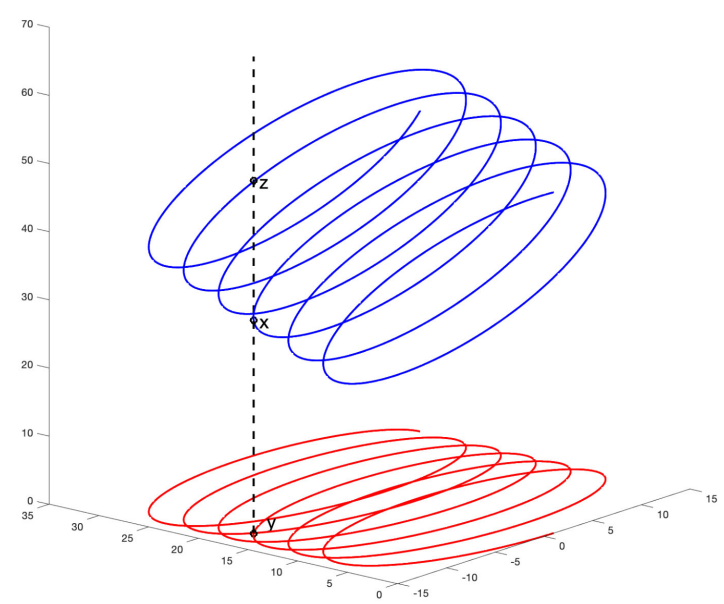

Fig. 2: Stable self-intersection of the projected trajectory

Then, if the first measurement corresponds to $y_{0}$, the system is not observable in the sense of Definition 1. Indeed, self-intersection means that there exists two points $t_{0} \neq s_{0}$ such that $h\left(F^{t_{0}}\left(\xi^{0}\right)\right)=h\left(F^{s_{0}}\left(\xi^{0}\right)\right)$ (we denote $x=F^{t_{0}}\left(\xi^{0}\right)$ and $z=F^{s_{0}}\left(\xi^{0}\right)$, $\xi_{0}$ being a point on the trajectory). Therefore, $P_{\Sigma}^{N}\left(\xi^{0}, t_{0}, \ldots\right)=P_{\Sigma}^{N}\left(\xi^{0}, s_{0}, \ldots\right)$ will never implies $F^{t_{0}}\left(\xi^{0}\right)=F^{s_{0}}\left(\xi^{0}\right)$ since $x \neq z$.

Remark 4: Il could be counterintuitive to obtain that the number of outputs should be greater than 3 instead of 2 in Theorem 3 (see Examples 1 and 2). Indeed, roughly speaking, if $p=2$, one can eliminate the time at each measurement and obtain the equivalent of a measurement in dimension 1 and one know ([7]) that system with one measurement and without control is generically observable. But one should 
consider that this result will make possible to reconstruct the trajectory, and not the state at the first measurement. This second property requires more information and could explain the need for $\mathrm{p}$ to be greater than 3 .

\section{CONCLUSION}

This article is a first attempt to pose the problem of observability (prior to the synthesis of observers) in the case of an unknown time stamp of the measurements. We have described a few examples mainly based on camera acquisition but the problem can arise in several other cases. We have in mind an application in robotics where we are able to determine some waypoints of the trajectory without knowing at what time the robot crossed these points.

We have proved the genericity of observability (in a sense adapted to the problem) when the number of outputs is greater or equal to 3 . It remains to study the observability of such systems in the presence of control, as well as to describe a canonical form of observable systems, in order to build convergent observers.

\section{REFERENCES}

[1] Ralph Abraham and Joel Robbin. Transversal Mappings and Flows. W. A. Benjamin, Inc., New York/Amsterdam, 1967.

[2] Sabeur Ammar, Majid Massaoud, and Jean-Claude Vivalda. Genericity of the strong observability for sampled systems. SIAM Journal on Control and Optimization, 56(2):1463-1490, jan 2018.

[3] Eric Busvelle. Observability of systems when measurements are not time-stamped. In 9th International Conference on Systems and Control. IEEE, Taylor \& Francis, 2021. Submitted.

[4] Éric Busvelle and Jean-Paul Gauthier. On determining unknown functions in differential systems, with an application to biological reactors. ESAIM: Control, Optimisation and Calculus of Variations, 9:509-551, aug 2003.

[5] Alireza Fatehi and Biao Huang. Kalman filtering approach to multirate information fusion in the presence of irregular sampling rate and variable measurement delay. Journal of Process Control, 53:15-25, 2017.

[6] Aïda Feddaoui, Nicolas Boizot, Eric Busvelle, and Vincent Hugel. High-gain extended kalman filter for continuous-discrete systems with asynchronous measurements. International Journal of Control, 93(8):2001-2014, 2020.

[7] Jean-Paul Gauthier and Ivan Kupka. Deterministic Observation Theory and Applications. Cambridge University Press, oct 2001.

[8] O. Hernández-González, M. Farza, T. Ménard, B. Targui, M. M’Saad, and C.M. Astorga-Zaragoza. A cascade observer for a class of mimo non uniformly observable systems with delayed sampled outputs. Systems \& Control Letters, 98:86-96, 2016.

[9] Morris Hirsch. Differential Topology, volume 33. Springer, 1997.

[10] Peng Shi. Filtering on sampled-data systems with parametric uncertainty. IEEE Transactions on Automatic Control, 43(7):1022-1027, 1998.

[11] Peng Shi. Filtering on sampled-data systems with parametric uncertainty. IEEE Transactions on Automatic Control, 43(7):1022-1027, 1998.

[12] Jean-Claude Vivalda. On the genericity of the observability of uncontrolled discrete nonlinear systems. In 2004 43rd IEEE Conference on Decision and Control (CDC) (IEEE Cat. No.04CH37601). IEEE, 2004.

[13] Huichai Zhang, Michael V Basin, and Mikhail Skliar. Itô-volterra optimal state estimation with continuous, multirate, randomly sampled, and delayed measurements. IEEE Transactions on Automatic Control, 52(3):401-416, 2007. 